\title{
Nurses Perception of Advance Nurse Practitioners Roles in Public Hospitals: A Qualitative Study
}

\author{
Manal Banaser ${ }^{1}\left(\mathbb{1}\right.$, Noora Al-Soqair ${ }^{2}$, Sara Al-Feher ${ }^{3}$ \\ ${ }^{1}$ Nursing Affairs General Department, MOH Agency for Therapeutic Services, Ministry of Health, Riyadh, Saudi, Arabia \\ ${ }^{2}$ Medical Assistant Services Outcome Administration, Al-Hasa Health Directorate, Ministry of Health, Saudi, Arabia \\ ${ }^{3}$ Nursing Clinical Support Department, Ministry of Health, King Saud Medical City, Riyadh, Saudi, Arabia \\ Email: mbanaser@moh.gov.sa
}

How to cite this paper: Banaser, M., Al-Soqair, N. and Al-Feher, S. (2021) Nurses Perception of Advance Nurse Practitioners Roles in Public Hospitals: A Qualitative Study. Open Journal of Nursing, 11, 513-527. https://doi.org/10.4236/ojn.2021.116044

Received: April 1, 2021

Accepted: June 26, 2021

Published: June 29, 2021

Copyright $\odot 2021$ by author(s) and Scientific Research Publishing Inc. This work is licensed under the Creative Commons Attribution International License (CC BY 4.0).

http://creativecommons.org/licenses/by/4.0/

\begin{abstract}
Aim: To explore the nurses' perceptions about the advanced nurses' practitioner's role in public hospitals in Saudi Arabia. Background: Advanced nurse practitioners are one of the leading professionals who contribute to health promotion, disease prevention, health counseling and patient education. However, misunderstandings of their roles may have an impact on the performances of the function that limits their access and the use of significant resources that can help patients to obtain treatment. Methods: This is a qualitative exploratory study conducted on nurses working in three public hospitals. Semi-structured interviews were conducted among fourteen nurses between October-January 2020. The three public hospitals were based in Eastern (1) and Central (2) Saudi Arabia regions. Data analysis was conducted through thematic analysis utilizing inductive and deductive coding. Results: The study found that the role of Advanced Practice Nurses will enhance, motivate, and sustain the status of nurses in Saudi Arabia. Findings indicated that advanced practitioners' educational preparedness, organizational, and contextual factors are important factors that need to be addressed to promote their role and to improve nurse's perception about the APN role in clinical practice. Conclusion: This research provided new evidence to support the need to raise understanding of the role of advanced nurse practitioners among nurse members, advanced nurses and clinical nurse specialists who wish to enhance quality healthcare delivery and job satisfaction in the Kingdom of Saudi Arabia. Recognizing the factors that comprise the function and status of APNs will assist health-care officials to make informed decisions when designing a national policy and a plan for the implementation and the development of the role of APNs.
\end{abstract}




\section{Keywords}

Advanced Nursing Practice, Role Preparedness, Nurses, Health System, Advanced Practitioners, Hospitals, Qualitative, Saudi Arabia

\section{Introduction}

Registered nurses with advanced education levels, experiences, skills, and knowledge can expand their role in the healthcare system by becoming Advanced Practice Nurses (APNs). They can diagnose, prescribe medications, and treat medical cases within established guidelines [1]. However, there are common barriers across countries that affect the implementation of APN roles in health settings. These barriers can be classified into either system-level or organizational-level barriers. The barriers at the system level include the absence of policy, guidelines, and regulations for the APN roles [2] [3]. Confusion about APN roles in healthcare settings, the scope of practice, use of different titles or terminology to describe nurse practitioners or advanced practice nurses, in addition to the absence of a recognized funding mechanism can impact the understanding of their role [4] [5] [6].

Implementing nurse practitioners' roles can decrease the need for general practitioners in primary care and improve patients' access to care. Nurse practitioners have been effective in the assessment and treatment of chronic disease, which decreases the need for family doctors [7]. Improved teamwork within a practice is another clinical benefit that can be achieved using nurse practitioners. There is an economic advantage to nurse practitioners because consultations with nurse practitioners are more cost-efficient than with a general practitioner [8]. Nurse practitioners can assist the healthcare system by administering care to patients with medical or complex or chronic conditions by providing medical and nursing care via their expanded scope of practice [9] [10] [11].

Advanced practice nursing roles aim to administer the best care to patients in the community and hospitals by improving their health within economically accountable methods. However, misunderstanding the APN role can affect the implementation of the role that borders their access and utilizing the significant resources, which can assist in administering care to patients in rural settings [2] [12]. Therefore, this research explores nurses, advanced practice nurses, and nurse leader perceptions of the APN role in Saudi Arabia.

\section{Background}

Advanced Practice Nursing (APN) roles in the United States could be traced back to the $19^{\text {th }}$ century when Sister Mary Bernard practiced as a nurse anesthetist in the 1870s. Nevertheless, nurse midwives practiced in that capacity since ancient times [13]. However, there are variations on the perspectives and the regulations of Advance Practice Nursing roles globally; therefore, the Interna- 
tional Council of Nurses (ICN, 2019) provided the following Definition facilitate a shared understanding and to guide more forth development recommending a master's degree as an entry-level:

"A Nurse Practitioner/Advanced Practice Nurse is a registered nurse who has acquired the expert knowledge base, complex decision-making skills, and clinical competencies for expanded practice, the characteristics of which are shaped by the context and/ or country in which s/ he is credentialed to practice." [14].

Furthermore, the ICN provides; educational, nature of practice, and regulatory mechanisms characteristics to distinguish advanced practice nursing roles. The educational features include; advanced-level educational preparation, formal recognition of the APN and Nurse Practitioner (NP) programs, and a formal system of licensure, registration, certification, and accreditation [14].

The recognition process of the advanced practice nursing roles differs among countries based on their education, legislation, accreditation, and healthcare systems demand. In the United States, the National Council of State Boards of Nursing (NCSBN) developed an advanced practice registered nurses (APRNs) Consensus Model to guide for state boards to unify the regulation of APRN roles [15]. The Consensus Model outlines the essential elements to regulate APRNs, which include; licensure, accreditation, certification, and education (LACE). Four leading roles were recognized, which include: certified nurse practitioner (CNP), clinical nurse specialist (CNS), certified nurse-midwife (CNM), and certified registered nurse anesthetist (CRNA). APRNs should be educated in one of the four roles and on the least one of the six population foci, which include: family/individual across the lifespan, psych/mental health, women's health/gender-related, adult-gerontology, neonatal, or pediatrics [15]. Passing a national certification exam and recertification in the APRN role and population is a requirement after finishing the post-graduate education in that role. Moreover, a license should be obtained to practice in one of the four aforementioned roles of APRN [15]. The title APRN is legally protected, and no one except those who are licensed to practice as APRN should use APRN title or any of its role titles [15].

In Canada, two of the APRNs roles are recognized, which are clinical nurse specialists (CNS), and the nurse practitioner (NP). However, due to the lack of national curriculum or consistent standard, APRN must meet the individual territorial or provincial nursing body requirements which generally include: a graduate education, passing the Canadian Nursing Association (CAN) nurse practitioner program exam, and registration with the appropriate province (Canadian Nursing Association [16]. There are legislation and regulations for the nurse practitioner role in all regions in Canada, and the title is protected. However, the clinical nurse specialist role is still lacking regulation, title protection, and only licensed as registered nurses [16].

The Definition of advanced nursing practice in Australia (AUS), however, is different. The Nursing and Midwifery Board of Australia (NMBA) identifies Advanced nursing practice (ANP) as a level of practice, not a role and is specific 
to the individuals within their context of practice whether they are enrolled nurses, registered nurses, or nurse practitioners [17]. Nurse practitioners, however, require a prescribed level of education, a specified advanced nursing practice experience, and continuous professional development, and the title is protected due to the additional legislative functions and the regulatory requirements [17]. Nurse practitioners were authorized to practice in New South Wales in 2000 with at least a master's degree as required by the low [18]. Similarly, in the United Kingdom (UK), the Royal College of Nursing (RCN) recognizes the advanced practice as a level of practice rather than a type of practice and recognizes different roles such as advanced nurse practitioner, advanced clinical practitioner, advanced clinical nurse specialist, and nurse consultants leading specialist services [19]. In the United Kingdom, the Advance Nursing Practice roles were established in a primary health care setting in 1981 [20]. Accreditation by endorsement is the primary recognized process in the UK and AUS.

Research reported that there are wide variations in educational requirements, the scope of practice, and regulations concerning the roles of advanced practice nursing across the globe [21]. A nurse practitioner's title varies from country to country and includes an advanced practice nurse, family nurse practitioner, and advanced nurse practitioner. Griffin and Melby in 2006 reported that only $11 \%$ of the participants indicated that they had a clear understanding of the role, while the other group concluded attitudes concerning the role were complex and multidimensional [22]. In 2013, a study conducted by Dalton reported similar findings [23]. Nurses reported a positive impact on the emerging role; however, concerns were raised regarding the erosion of the traditional nurse role, increased risk of litigation and acceptance from medical colleagues, and medical staff was equally concerned that nurse practitioners would "cherry-pick" patients, leaving the more mundane tasks to the medical staff [23].

The healthcare system in Saudi Arabia has been given "high priority" in the government. Improving and developing the healthcare system in Saudi Arabia was clear in terms of quality and quantity of services in the past few years, included the availability of high levels of care providers to the community [24]. The Ministry of Health (MOH) in Saudi Arabia is supporting the public healthcare system, which means the government provides all the citizens and expatriates free access to all public healthcare facilities. The responsibility for $\mathrm{MOH}$ in Saudi Arabia is to manage, plan, formulate health policies, supervise health programs, and monitor health services in the private facilities [24] [25].

Many countries are considering improving their healthcare systems by increasing the efficiency of healthcare delivery [26]. Healthcare systems can be improved through new or more advanced roles for health professionals. Nurses are one group of clinical professionals who can assist in optimizing healthcare delivery in health settings. Given the growing demands of healthcare systems, nurses can increase access to care in the face of a shortage or a limited number of doctors [27]. Nurses are the largest profession of healthcare providers in health 
settings and the primary profession with patient contact [28]. According to Almalki et al. (2011), nurses in Saudi Arabia constitute 50.3\% of the health workforce in the $\mathrm{MOH}$ [24]. Few previous researches in the form of review or discussion papers discussed the need to integrate the role of advanced nursing practice in Saudi health care settings [11] [29]. Although there are many advanced nursing program graduates working in public hospitals, there is a dearth of literature considering the advanced nursing practice role. Additionally, legalization and regulatory support for advanced nursing practice is still underway. This study therefore conducted to assess and explore the knowledge and perception of APN's position among nurses in Saudi Arabia.

\subsection{Aims}

The research aims to explore the perception of nurses, advanced practice nurses, and nurse leaders about the role of advanced practice nurses in Saudi public hospital settings.

\subsection{Research Questions}

- How do nurses, nurse leaders, advanced nurse practice graduates perceive the ANP role in the Saudi public hospital settings?

- What are the recommendations for facilitating the APN role in Saudi healthcare settings?

\section{Methods}

\subsection{Design}

This study used a qualitative exploratory design with semi-structured interviews.

\subsection{Sample/Participants}

A purposive sample selected from the Eastern and Central regions in Saudi Arabia. Purposive sampling of nurses in three $\mathrm{MOH}$ public hospitals in Riyadh and Eastern regions was employed. Fourteen nurses included advanced practice nursing master graduates and clinical nurse specialists, and nurse directors participated in audiotaped semi-structured interviews. The sample included six nurses holding an APN master's degree, six clinical nurse specialists holding a master's degree in a nursing specialty, and four nurse leaders.

\subsection{Setting}

Three large public (300 beds \& above) hospitals are involved, two from Riyadh region and one from the Eastern region. King Salman hospital (Riyadh), Imam Abdurrahman bin Faisal hospital (Riyadh), and King Fahd general hospital (ALHASA). These settings were chosen based on the multi-center research design in order to reflect broader demographic features, as well as the Kingdom's restricted supply of nurses who graduated from advanced practice nursing programs. 


\subsection{Ethical Considerations}

Ethical approvals are granted from the research ethics committee at the General Directorate of research in MOH (IRB/2019-158M). Participants offered the right to reject responding to the questions or withdraw at any time. Further, researchers explained to participants that all providing information is kept confidential with anonymity and privacy.

\subsection{Recruitment Procedure}

After ethical approval was obtained, invitation letters and information sheets about study purposes were sent by electronic mail communication to nurses working in the three public hospitals at $\mathrm{MOH}$. Participants who agreed to participate were sent booking arrangements for an interview date, time, and place. The interview procedure explained to the interviewee stating that there is no risk in participating and that they can withdraw at any time. Confidentiality of the data assured, and that the information is only used for research purposes.

\subsection{Data Collection}

Data were collected by semi-structured interviews. The co-researchers interviewed participants in the English language between December 2019 and January 2020. Most of the interviews $(n=14)$ were conducted face to face in Riyadh and Alhassa Region. A total of 14 nurses agreed to participate in this research. Due to nurses' circumstances of busy schedules, some interviews were held by telephone. Eight telephones skype and six face to face interviews were conducted. The interviews conducted by the co researchers in meeting room in their workplace. The participants appeared comfortable, reflective, and ready to discuss the research topic.

Participants were four nursing leaders holding a master's degree, six staff nurses holding a master's degree, and four advanced practice nurses. The researchers interviewed the participants and audiotaped interview sessions after their permission. Instances of the interview questions are exhibited in Table 1. Additionally, the researchers asked the participants' probing questions to explain the meaning and inspire a superior comprehension of their views. Interview time ranged from 30 to $60 \mathrm{~min}$.

Table 1. Example of interview questions.

Interview questions schedule

1) What do you know about the APN role?

2) What is the educational preparation for advanced practice nurses in Saudi?

3) What is the benefit of implementing the APN role in health settings?

4) What are the various practice settings in which the nurse can exhibit the role?

5) Describe the nature of nurse prescribing, and do you think APN nurses can prescribe?

6) How do you see the APN role in promoting health services in Saudi Arabia? 


\subsection{Data Analysis}

The analysis conducted through thematic analysis utilizing inductive and deductive coding. Analysis of the interview transcripts was encouraged by the organization of emerged themes into NVIVO 10 software. The NVIVO is the approved electronic software for organizing data from qualitative research. Thematic analysis followed the six phases distinguished by Fereday and Muir-Cochrane [30]. Initially, the coding manual was created by co-researchers on exploration questions. Instead, all researchers tested the coding. Comprehension of coding was generally high, and the few minor issues that emerged were discussed and settled before continuing the analysis. The analysis includes inductive coding from explained removes; this assists in distinguished new themes. The coded themes were verified and legitimized as being illustrative of the first information by returning to the content from the first interview separates. Subsequently, major themes that captured the views of nurse leaders, clinical nurse practitioners, and advanced nurse practice on advanced nurse roles were created.

\section{Results}

Three central themes were distinguished, alongside with the related subthemes (Table 2). The primary theme exhibits the nurses' perceptions of ANP's role within the subthemes that include education, independence, prescribing drugs, primary, acute and specialized healthcare. While the second theme explores the preparedness of the ANP role, which includes the certificate and experiences. The third theme discusses the organizational factors and benefits of ANP role implementation, and the subtheme includes the patients' outcomes and how it could improve health care services, and the cultural influences of the ANP role in the Saudi context.

\subsection{Nurse's Perception of APN Role}

The theme of education among two of the 14 participants emerged. There was little understanding of the role of advanced nurses. However, participants generally perceived the advanced nurse should have the skills and high-level knowledge of advanced clinical competencies and experience based on evidence.

- Update her knowledge and skills following Evidence Based Practice (Participant 3$)$.

- Use of best practices and evidence-based care to achieve outcomes (Participant 6).

Table 2. Key themes from qualitative research.

\begin{tabular}{ll}
\hline Themes & Subthemes \\
\hline Nurse's perception of APN role & $\begin{array}{l}\text { Education, independence, prescribing drugs, } \\
\text { Primary, acute, and specialized healthcare. }\end{array}$ \\
Nurse preparedness of APN role & $\begin{array}{l}\text { Educational qualification, experience, no information, } \\
\text { Organizational Factors }\end{array}$ \\
$\begin{array}{l}\text { Patients' outcomes, improve health care services, promoting } \\
\text { health services, cultural factors }\end{array}$ \\
\hline
\end{tabular}


Many participants indicated that the advanced nurses would have the expertise in diagnosis, assessment, research, and advanced treatment, which is after a high-level clinical study that involves certain specialties such as organology and pharmacology so that they have the skills and ability to take appropriate procedures and nursing care.

- They have more skills and knowledge in assessment and physical examination, pathophysiology, and pharmacology, and they have the authority to diagnose and prescribe medications (Participant 7 ).

Independence and responsibility for inpatient care are stated specifically and often implicitly by some participants that a decision was taken on treatments, medical strategies, and nursing care. They mentioned that advanced nurses made decisions about the patient with complete and high independence without referring to the doctor. APN is more capable of functioning independently in various healthcare environments and can treat, assess, and prescribe medicines.

- They are nurses who complete the APN program after BSN. They have more skills and knowledge in assessment and physical examination, pathophysiology, and pharmacology, and they have the authority to diagnose and prescribe medications (Participant 7).

- The APN prescribes, orders, and implements interventions and treatments identified in the plan of care, including prescribing pharmacologic treatments (Participant 8).

The prescription of the medicines indicated in an explicit statement by the three participants holding two baccalaureate and one master's degrees in nursing, whereas it came only once from one of the research participants holding a master's degree in advanced practice nursing and they all mentioned that this is one of the tasks of the advanced practice nurse, which is an indiscriminate matter.

\subsection{Nurse Preparedness of APN Role}

When participants were asked about the educational preparation of nurses to be advanced practitioners, most participants assured that the education level could range from the baccalaureate to the master's level in a nursing specialty.

One participant mentioned the educational preparation of the advanced practice nurse through their continuing education, which requires an advanced practice exercise a higher level of education by first obtaining a Master of Science in Nursing (MSN) at least and the participants also mentioned some specialties related to the advanced practitioner such as a nurse practitioner:

"Advanced nurse practitioners may specialize in different areas. For instance, Certified Nurse Anesthesiologist (CRNA) and Clinical Nurse Specialist (CNS) provides specialized care in several areas: cardiology, oncology, neonatal diseases, gynecology, obstetrics, pediatrics, neurology and mental health-nurse" (Participant 11).

One of the participants mentioned that she joined a recent advanced nursing 
program in Saudi Arabia.

$I$ would be an advanced nurse practitioner by obtaining a master's degree in advanced nursing practice that started on September 17th, 2017, at Princess Norah University. It is a full-time semester program offered for four semesters including lectures, skills, and clinical practices that in turn seeks to integrate theory and practice and provide nurses with the knowledge and skills necessary to advance in their practice and empower them to provide safe and high-quality care (Participant 12).

Whereas one of the participants mentioned that APN should be prepared by a master with no less than 5 to 6 years of work experience. Also, work in the acute to the medium-sized large-care hospital. So, she can do the tasks of the advanced practitioner. However, two of the participants, stated that they did not know that there was even a university in the Kingdom of Saudi Arabia that offered a program or certificate for nursing specialization to work as an advanced practicing nurse.

"I didn't know there is an educational program for advance nurse practitioners in Universities at the Kingdom" (Participant 5).

\subsection{Organizational Factors}

The subtheme prompting health services in Saudi Arabia was questioned by the researcher to the participants to identify their expectation of implementing the APN role in Saudi Arabia and its impacts on promoting health services. Nurses' leaders and nurses assumed the APN role could build up the health services and increase the awareness of the importance of nursing.

As one nurse illustrates:

"The ANP will establish a new generation of nurses' knowledge, skills, and high self-confidence" (Participant 10).

Remarkably, some participants viewed nurses' cultural perception, such as the image of nursing, as this may positively impact the public view of those who work as nursing practitioners.

Additionally, the leaders believed they can provide a high quality of care and assist in early detection of serious health problems in primary health care and refer the patients to the tertiary health settings. As one of the nurse leaders said:

"The ANP could treat the whole patient, not just the disease" (Participants 9)

However, few of the participants answered as:

"No idea yet actually because I did not see it practiced in the MOH hospital" (Participant 8).

However, ANP participants found their role is affective and can assist in promoting health, especially in primary health care. One ANP said,

"They can cover the shortage of physicians in primary health care, mainly in remote and rural areas. They can improve access to health services and reduce the length of waiting time" (Participant 12).

Thus, APN has a positive impact on health care services in developed coun- 
tries proved a significant improvement level of health care services (Woo et al., 2019). APN's role from the APN perspective in Saudi Arabia can improve the quality of care and career prospects for nurses.

\section{Discussion}

This is an important first study to explore the opinions of nursing leaders and those holding degrees in advanced practice nursing in some public hospitals in the Kingdom of Saudi Arabia. Qualitative results help reduce the gap between reality and the aspirant of the role of the advanced nurse practitioner who is not subject previously to research. It also provides a great insight into the knowledge of registered nursing and advanced practice nursing, the role that the advanced practitioner nurse plays, and the influence of experience and educational backgrounds on the principal tasks of the practitioner.

Where it was internationally clear that there is confusion between knowledge about the role of the practitioner and those who are unaware of a different role to that of the registered nurse [2], the study also found that there is a discrepancy in the view of some participants who are familiar with the profession of nursing between the expertise of practice and education when specifying the requirements that the nurse must obtain to be able to test, prescribe and possess the competencies and the specialized professional knowledge based on evidence. Nevertheless, in an international context, Nurse practitioners, however, require a prescribed level of education, a specified advanced nursing practice experience, and continuous professional development, and the title is protected due to the additional legislative functions and the regulatory requirements [3] [18].

In this finding, when we address the requirements for obtaining the name of the advanced practice nurse, we find that there are differences in educational requirements and professional practice from the responses of all participants. Moreover, there are no classification and regulations for the tasks or roles of advanced practice nursing in the Kingdom of Saudi Arabia, although the presence of Princess Nora bent Abdul Rahman University and scholarships abroad carrying this specialty.

Similarly, the clinical nurse specialist role is still lacking regulation, title protection, and only licensed as registered nurses [31]. As for the benefit of applying the role of clinical nursing in improving healthcare quality, all the participants responded that multiple benefits occur when activating the role of the APN.

This is what was mentioned in the introduction to this paper that there is an improvement in the health care provided and also an improvement in the economic aspect, and since nursing is one of the most important basic professions in the world and with a shortage of doctors, it was important to improve their health care systems by increasing the efficiency of health care delivery by expanding nursing roles, which in turn reduces the need for general practitioners in primary care and improves patients' access to care. Implementing nurse practitioners' roles can decrease the need for general practitioners in primary care 
and improve patients' access to care. Nurse practitioners have been effective in the assessment and treatment of chronic disease, which decreases the need for family doctors [7].

The findings show that some organizational factors, including lack of policy, guidance, and regulation on APN roles, represent barriers at the system level. Similarly, previous studies indicated that confusion about APN roles in healthcare settings, the scope of practice, use of different titles or terminology to describe nurse practitioners or advanced practice nurses, in addition to the absence of a recognized funding mechanism can impact the understanding of their role [4] [32].

Interestingly, participants also mention cultural factors that have a significant impact on understanding the perception of the role of advanced practitioners. Findings suggested that public awareness of nursing image and family and religious influences would be captivated and could have an impact on the role of advanced nurses in Saudi Arabia. Accordingly, research suggested that the social image of nursing could affect the promotion of the nurse-patient relationship and thereby affect the advance role development of nurses [3]. Conversely, previous research indicated that implementing an advanced nursing role may improve the public perception and increase the nursing profession's reputation [33]. Moreover, the disclosure of information and the doctor-power dynamics inherited previously from the care delivery model are equally important factors that could still have an impact on the implementation of the role of advanced nurses in Saudi health care [34]. However, given the new integrated model of a patient-centered care in the healthcare in Saudi Arabia as part of the Ministry of Health Transformation and Health Vision 2030 [35], these cultural barriers can be addressed in the future through strategic objectives that could improve the wise use of healthcare delivery system resources by integrating the role of advanced nurse practitioners in healthcare practice.

\section{Limitation of the Study and Future Research}

This qualitative research has explored nurses' understanding of the role of the advanced practitioner in Saudi Arabia. While this research gives further insight into how nurses perceive the role of advanced practitioners and what factors influence this role. Future research needs to consider involving physicians that could add more insights to understand how well-integrated health care system can be widely used as the best-advanced nurse role. Further research needs an in-depth exploration of the cultural factors that underpin the implementation of their role, which can add in-depth insights into the findings and thus enforce an effective change to promote advanced nurses' practice.

\section{Conclusion}

This research provided new evidence to support the need to raise understanding of the role of advanced nurse practitioners among nurse members, advanced 
nurses and clinical nurse specialists who wish to enhance quality healthcare delivery and job satisfaction in the Kingdom of Saudi Arabia. Therefore, advanced practitioners' professional preparedness, organizational, and contextual factors are important factors that need to be addressed to promote their role in clinical practice. Recognizing these factors that influenced the function and status of APNs will allow health-care policymakers to make informed decisions when developing a national policy and strategy for implementation and development of the role APNs.

\section{Implication for Practice}

To improve the quality of care, patient safety, and job satisfaction, policymakers and nursing authorities must, therefore, provide regulatory support for the autonomous role of advanced practitioners before their implementation in practice. Further steps to authorize, legalize ethically and provide their scope of practice are paramount in building up their professional entities in the advancement of health care. Moreover, key nursing authorities should raise the awareness and enhance the perception of advanced nurse practitioners' role definition among nursing members and hospital directors to ensure wide health system acceptance. These findings can be trans-cultured into other international contexts seeking to incorporate the role and preparation of advanced practitioners in advancing clinical practice.

\section{Acknowledgements}

The authors would like to express their sincere gratitude to the General Department of Nursing at the $\mathrm{MOH}$ Agency for therapeutic services for the great support to this paper as one of the projects of the researcher capability building project (BAHITH 2019). The authors are also grateful to all the nurses who participated in this study.

\section{Conflicts of Interest}

The authors have declared no conflict of interests.

\section{References}

[1] Reay, T., Patterson, E.M., Halma, L. and Steed, W.B. (2006) Introducing a Nurse Practitioner: Experiences in a Rural Alberta Family Practice Clinic. Canadian Journal of Rural Medicine, 11, 101-107. https://pubmed.ncbi.nlm.nih.gov/16630436/

[2] Fealy, G.M., Casey, M., O’Leary, D.F., McNamara, M.S., O’Brien, D., O’Connor, L., Rita, S. and Stokes, D. (2018) Developing and Sustaining Specialist and Advanced Practice Roles in Nursing and Midwifery: A Discourse on Enablers and Barriers. Journal of Clinical Nursing, 27, 3797-3809. https://doi.org/10.1111/jocn.14550

[3] Jokiniemi, K., Suutarla, A., Meretoja, R., Kotila, J., Axelin, A., Flinkman, M., Heikkinen, K. and Fagerström, L. (2020) Evidence-Informed Policymaking: Modelling Nurses' Career Pathway from Registered Nurse to an Advanced Practice Nurse. International Journal of Nursing Practice, 26, e12777. https://doi.org/10.1111/ijn.12777 
[4] Bryant-Lukosius, D. and Dicenso, A. (2004) A Framework for the Introduction and Evaluation of Advanced Practice Nursing Roles. Journal of Advanced Nursing, 48, 530-540. https://doi.org/10.1111/j.1365-2648.2004.03235.x

[5] Lamb, A., Martin-Misener, R., Bryant-Lukosius, D. and Latimer, M. (2018) Describing the Leadership Capabilities of Advanced Practice Nurses Using a Qualitative Descriptive Study. Nursing Open, 5, 400-413. https://doi.org/10.1002/nop2.150

[6] Gillingham, I., Neubeck, L., Williams, B. and Dawkes, S. (2020) Role Expansion for Nurses in the Cardiac Catheter Laboratory: Findings from a Systematic Scoping Review. British Journal of Cardiac Nursing, 15, 1-14.

https://doi.org/10.12968/bjca.2019.0128

[7] Bonsall, K. and Cheater, F.M. (2008) What Is the Impact of Advanced Primary Care Nursing Roles on Patients, Nurses, and Their Colleagues? A literature Review. International Journal of Nursing Studies, 45, 1090-1102.

https://doi.org/10.1016/j.ijnurstu.2007.07.013

[8] Hu, J. and Forgeron, P. (2018) Thinking, Educating, Acting: Developing Advanced Practice Nursing. International Journal of Nursing Sciences, 5, 99-100. https://doi.org/10.1016/j.ijnss.2018.04.006

[9] King, J., Corter, A., Brewerton, R. and Watts, I. (2012) Nurse Practitioners in Primary Care: Benefits for Your Practice. Canberra: Australian General Practice Network, Julian King \& Associates Ltd., Auckland.

[10] Kilpatrick, K., Lavoie-Tremblay, M., Ritchie, J.A., Lamothe, L. and Doran, D. (2012) Boundary Work and the Introduction of Acute Care Nurse Practitioners in Healthcare Teams. Journal of Advanced Nursing, 68, 1504-1515. https://doi.org/10.1111/j.1365-2648.2011.05895.x

[11] Hibbert, D., Aboshaiqah, A.E., Sienko, K.A., Forestell, D., Harb, A.W., Yousuf, S.A., Kelley, P.W., Brennan, P.F., Serrant, L. and Leary, A. (2017) Advancing Nursing Practice: The Emergence of the Role of Advanced Practice Nurse in Saudi Arabia. Annals of Saudi Medicine, 37, 72-78. https://doi.org/10.5144/0256-4947.2017.72

[12] Echeverri, R. (2006) Nurse Practitioners of Advanced Practice Nursing: Roles, Influence, and Outcomes. Clinical Nurse Specialist. The International Journal for Advanced Nursing Practice, 20, 154. https://doi.org/10.1097/00002800-200605000-00015

[13] Gray, M., Ratliff, C. and Mawyer, R. (2000) A Brief History of Advanced Practice Nursing and Its Implications for WOC Advanced Nursing Practice. Journal of Wound, Ostomy and Continence Nursing, 27, 48-54. https://doi.org/10.1097/00152192-200001000-00019

[14] International Council of Nurses (2020) Guidelines on Advanced Practice Nursing. https://www.icn.ch/system/files/documents/2020-04/ICN APN\%20Report EN WE B.pdf

[15] The Advanced Practice Registered Nurses Consensus Work Group \& The National Council of State Boards of Nursing (2008) Consensus Model for APRN Regulation: Licensure, Accreditation, Certification \& Education. https://www.ncsbn.org/Consensus Model for_APRN_Regulation_July 2008.pdf

[16] Canadian Nurses Association (2019) Advanced Practice Nursing. https://cna-aiic.ca/en/nursing-practice/the-practice-of-nursing/advanced-nursing-p $\underline{\text { ractice }}$

[17] Nursing and Midwifery Board of Australia (NMBA) (2017) Guidelines: For Nurses Applying for Endorsement as a Nurse Practitioner.

https://www.nursingmidwiferyboard.gov.au/Codes-Guidelines-Statements/Professi 
onal-standards/nurse-practitioner-standards-of-practice.aspx

[18] Helms, C., Gardner, A. and McInnes, E. (2017) Consensus on an Australian Nurse Practitioner Specialty Framework Using Delphi Methodology: Results from the CLLEVER 2 Study. Journal of Advanced Nursing, 73, 433-447. https://doi.org/10.1111/jan.13109

[19] Royal College of Nursing (2018) Advanced Level Nursing Practice: Introduction. https://www.rcn.org.uk/professional-development/publications/pub-006894

[20] Bowling, A. and Stilwell, B., Eds. (1988) The Nurse in Family Practice: Practice Nurses and Nurse Practitioners in Primary Health Care. Scutari Press, London, 70.

[21] Heale, R. and Rieck, B.C. (2015) An International Perspective of Advanced Practice Nursing Regulation. International Nursing Review, 62, 421-429. https://doi.org/10.1111/inr.12193

[22] Griffin, M. and Melby, V. (2006) Developing an Advanced Nurse Practitioner Service in Emergency Care: Attitudes of Nurses and Doctors. Journal of Advanced Nursing, 56, 292-301. https://doi.org/10.1111/j.1365-2648.2006.04025.x

[23] Dalton, M.A. (2013) Perceptions of the Advanced Nurse Practitioner Role in a Hospital Setting. British Journal of Nursing, 22, 48-53. https://doi.org/10.12968/bjon.2013.22.1.48

[24] Almalki, M., FitzGerald, G. and Clark, M. (2011) The Nursing Profession in Saudi Arabia: An Overview. International Nursing Review, 58, 304-311. https://doi.org/10.1111/j.1466-7657.2011.00890.x

[25] Al-Dossary, R.N. (2018) The Saudi Arabian 2030 Vision and the Nursing Profession: The Way Forward. International Nursing Review, 65, 484-490. https://doi.org/10.1111/inr.12458

[26] Hill, M.N., Parker, J., Liu, H., Hu, Y. and Guo, G. (2017) Strategic Directions and Actions for Advanced Practice Nursing in China. International Journal of Nursing Sciences, 4, 8-11. https://doi.org/10.1016/j.ijnss.2016.12.010

[27] Aljohani, K.A.S. (2020) Nursing Education in Saudi Arabia: History and Development. Cureus, 12, e7874. https://dx.doi.org/10.7759\%2Fcureus.7874

[28] Naylor, M.D. and Kurtzman, E.T. (2010) The Role of Nurse Practitioners in Reinventing Primary Care. Health Affairs (Millwood), 29, 893-899. https://doi.org/10.1377/hlthaff.2010.0440

[29] Albejaidi, F. and Nair, K.S. (2019) Building the Health Workforce: Saudi Arabia's Challenges in Achieving Vision 2030. The International Journal of Health Planning and Management, 34, e1405-e1416. https://doi.org/10.1002/hpm.2861

[30] Fereday, J. and Muir-Cochrane, E. (2006) Demonstrating Rigor Using Thematic Analysis: A Hybrid Approach of Inductive and Deductive Coding and Theme Development. International Journal of Qualitative Methods, 5, 80-92. https://doi.org/10.1177/160940690600500107

[31] Alotaibi, T. and Al Anizi, A. (2019) Literature Review on Comparisons of Advanced Clinical Nurse Practitioner Role in Saudi Arabia and United Kingdom. Journal of Radiology Nursing, 38, 264-271. https://doi.org/10.1016/j.jradnu.2019.09.007

[32] Sullivan, J.A., Dachelet, C.Z., Sultz, H.A., Henry, M. and Carrol, H.D. (1978) Overcoming Barriers to the Employment and Utilization of the Nurse Practitioner. American Journal of Public Health, 68, 1097-1103. https://doi.org/10.2105/AJPH.68.11.1097

[33] Brigitte, F.Y.W., Zhou, W., Lim, T.W. and Tam, W.W.S. (2019) Practice Patterns and Role Perception of Advanced Practice Nurses: A Nationwide Cross-Sectional 
Study. Journal of Nursing Management, 27, 992-1004.

https://doi.org/10.1111/jonm.12759

[34] Banaser, M. Kathleen, S. and Nicola, C. (2017) A Qualitative Study of Patient Satisfaction in Oncology Wards Setting in Saudi Arabia. Research and Reviews: Journal of Nursing and Health Sciences, 3, 85-97.

[35] Al-Hanawi, M.K., Khan, S.A. and Al-Borie, H.M. (2019) Healthcare Human Resource Development in Saudi Arabia: Emerging Challenges and Opportunities-A Critical Review. Public Health Reviews, 40, Article No. 1.

https://doi.org/10.1186/s40985-019-0112-4 